\title{
Pseudomonas aeruginosa $\beta$-lactamase induction requires two permeases, AmpG and AmpP
}

\author{
Kok-Fai Kong ${ }^{1 *}$, Alian Aguila ${ }^{1}$, Lisa Schneper ${ }^{2}$, Kalai Mathee ${ }^{2^{*}}$
}

\begin{abstract}
Background: In Enterobacteriaceae, $\beta$-lactam antibiotic resistance involves murein recycling intermediates. Murein recycling is a complex process with discrete steps taking place in the periplasm and the cytoplasm. The AmpG permease is critical to this process as it transports $\mathrm{N}$-acetylglucosamine anhydrous $\mathrm{N}$-acetylmuramyl peptides across the inner membrane. In Pseudomonadaceae, this intrinsic mechanism remains to be elucidated. Since the mechanism involves two cellular compartments, the characterization of transporters is crucial to establish the link.

Results: Pseudomonas aeruginosa PAO1 has two ampG paralogs, PA4218 (ampP) and PA4393 (ampG). Topology analysis using $\beta$-galactosidase and alkaline phosphatase fusions indicates ampP and ampG encode proteins which possess 10 and 14 transmembrane helices, respectively, that could potentially transport substrates. Both ampP and ampG are required for maximum expression of $\beta$-lactamase, but complementation and kinetic experiments suggest they act independently to play different roles. Mutation of ampG affects resistance to a subset of $\beta$-lactam antibiotics. Low-levels of $\beta$-lactamase induction occur independently of either ampP or ampG. Both ampG and ampP are the second members of two independent two-gene operons. Analysis of the ampG and ampP operon expression using $\beta$-galactosidase transcriptional fusions showed that in PAO1, ampG operon expression is $\beta$-lactam and $a m p R$-independent, while ampP operon expression is $\beta$-lactam and ampR-dependent. $\beta$-lactam-dependent expression of the ampP operon and independent expression of the ampG operon is also dependent upon ampP.

Conclusions: In P. aeruginosa, $\beta$-lactamase induction occurs in at least three ways, induction at low $\beta$-lactam concentrations by an as yet uncharacterized pathway, at intermediate concentrations by an ampP and ampG dependent pathway, and at high concentrations where although both ampP and ampG play a role, ampG may be of greater importance. Both $a m p P$ and $a m p G$ are required for maximum induction. Similar to $a m p C$, $a m p P$ expression is inducible in an ampR-dependent manner. Importantly, ampP expression is autoregulated and ampP also regulates expression of ampG. Both AmpG and AmpP have topologies consistent with functions in transport. Together, these data suggest that the mechanism of $\beta$-lactam resistance of $P$. aeruginosa is distinct from well characterized systems in Enterobacteriaceae and involves a highly complicated interaction between these putative permeases and known Amp proteins.
\end{abstract}

\section{Background}

Pseudomonas aeruginosa is a Gram negative opportunistic pathogen. As a frequent colonizer of catheters and the most frequent fatal causative agent of ventilatorassisted pneumonia, it is one of the most common agents in health-care associated infection [1]. Lung

\footnotetext{
* Correspondence: concordeflight@gmail.com; Kalai.Mathee@fiu.edu 'Department of Biological Sciences, College of Arts and Sciences, Florida International University, Miami, FL USA

${ }^{2}$ Department of Molecular Microbiology and Infectious Diseases, Herbert Wertheim College of Medicine, Florida International University, Miami, FL USA

Full list of author information is available at the end of the article
}

deterioration due to chronic infection by $P$. aeruginosa affects patients with chronic obstructive pulmonary disorder and is a leading cause of morbidity and mortality in cystic fibrosis patients [2]. P. aeruginosa infection treatment is often difficult because of the organism's intrinsic and acquired antibiotic resistance. This is due to the presence of multidrug efflux pumps [3], low outer membrane permeability [4], hypermutability [5], biofilm formation [6], and $\beta$-lactamase expression $[7,8]$.

$P$. aeruginosa has two chromosomally encoded $\beta$-lactamases: the PoxB oxacillinase and the AmpC cephalosporinase [8-10]. Much of what is known about
C Biomed Central

() 2010 Kong et al; licensee BioMed Central Ltd. This is an Open Access article distributed under the terms of the Creative Commons Attribution License (http://creativecommons.org/licenses/by/2.0), which permits unrestricted use, distribution, and reproduction in any medium, provided the original work is properly cited. 
AmpC regulation is from studies in Escherichia coli, Citrobacter freundii and Enterobacter cloacae. These studies have elegantly demonstrated that induction of AmpC, the chromosomal $\beta$-lactamase, involves $a m p R$, $a m p D$, and $\operatorname{amp} G$, encoding a LysR type transcriptional factor, an amidase, and a permease, respectively [11].

Expression of C. freundi AmpR in E. coli revealed that during normal physiological growth, AmpR, in the presence of UDP-MurNAc-peptide, binds to the $a m p C$ promoter and inhibits expression [12]. In E. coli, the addition of $\beta$-lactam antibiotics causes an increase in the cytosolic 1,6-anhydro- $\mathrm{N}$-acetylmuramyl-L-Ala$\gamma$-D-Glu-meso-diaminopimelic acid (anhMurNAc-tripeptide) concentration, and a decrease in the cytosolic UDP-N-acetylmuramyl-L-Ala- $\gamma$-D-Glu-meso-DAPD-Ala-D-Ala (UDP-MurNAc-pentapeptide) [12]. It was postulated that AmpR can either activate or repress transcription from the $a m p C$ promoter and that its activity is dependent upon the nature of the bound effector molecule. In vitro, in the presence of UDPMurNAc-pentapeptide, AmpR represses transcription of $a m p C$, whereas in the presence of 1,6-anhMurNActripeptide, AmpR activates $a m p C$ [12]. Thus, it is postulated that binding of 1,6-anhMurNAc-tripeptide alters the conformation of AmpR from the repressive to the activating mode, facilitating the expression of ampC [12]. High-levels of 1,6-anhMurNAc-tripeptide accumulate in the absence of $a m p D$. AmpD is an amidase that cleaves 1,6-anhMurNAc-tripeptide [13]. Induction of E. cloacae ampC was also shown to be amp G-dependent [14]. $\beta$-lactamase fusion analysis suggests that E. coli AmpG contains 10 transmembrane segments and two large cytoplasmic loops [15]. E. coli AmpG was shown to transport $\mathrm{N}$-acetylglucosamineanhydrous $\mathrm{N}$-acetylmuramic acid (GlcNAc-anhMurNAc) and GlcNAc-anhMurNAc-tri, -tetra, and -pentapeptides $[16,17]$.

Comprehensive and elegant studies using Enterobacteriaceae established the paradigm of the $\beta$-lactamase induction mechanism. Orthologs of $a m p R$, $a m p D$, and amp $G$ are found in numerous Gram-negative species [18]. Whether similar mechanisms are employed in all these organisms has not been established. It is possible that the induction mechanism could differ. The $\beta$-lactamase induction mechanism of $P$. aeruginosa has not been well-defined; however, it is known that $P$. aeruginosa AmpR regulates expression of $\operatorname{ampC}$ as in other organisms [8-10]. Similar to other systems, $a m p R$ is located upstream of the $a m p C$ gene [10]. Additionally, $P$. aeruginosa AmpR controls transcription of the oxacillinase, $p \circ x B$, and several genes involved in virulence [8-10]. Loss of AmpR in P. aeruginosa causes a significant elevation in $\beta$-lactamase activity and other virulence factors [10]. P. aeruginosa also differs from other previously studied systems in that its genome has two ampG orthologs, PA4218 and PA4393 [19]. The current study reveals that these two genes, $P A 4218$ and $P A 4393$, are required for $\beta$-lactamase induction, hence they have been named $\operatorname{amp} P$ and $\operatorname{amp} G$, respectively. Consistent with their putative roles as permeases, fusion analysis suggests that AmpG and AmpP have 14 and 10 transmembrane helices, respectively. Expression of ampP is dependent upon AmpR and is autoregulated. Together, these data suggest the distinctiveness of $P$. aeruginosa $\beta$-lactamase induction, as it is the first system that potentially involves two permease paralogs, and contribute to the general understanding of the induction mechanism.

\section{Results}

\section{Genome Sequence Analysis of the PA4218 and PA4393 Operons}

E. coli AmpG has been shown to be a permease that transports GlcNAc-anhMurNAc peptides from the periplasm to the cytoplasm [13,17]; however, the AmpG function in $P$. aeruginosa has not been described. BLAST analysis of the E. coli AmpG sequence against the sixframe translation of the PAO1 genome identified two open reading frames, $P A 4218$ and $P A 4393$, with significant homology $[20,21]$. Global alignment using the NeedlemanWusch algorithm [22] demonstrated that PA4218 is $21.8 \%$ identical and $34.8 \%$ similar, while PA4393 is $23.2 \%$ identical and 34.3\% similar to AmpG (Figure 1). The Pseudomonas Genome Database identifies PA4393 as encoding a putative permease with an alternate name of $a m p G$, while PA4218 is identified as encoding a probable transporter [23]. Thus, $P A 4393$ will be referred to as $a m p G$ and $P A 4218$ as $\operatorname{amp} P$ (P for permease).

Analysis of the sequences around ampG and ampP revealed that they were in close proximity to two respective upstream ORFs. Based upon sequence analysis, it is likely that $\operatorname{amp} G$ and $\operatorname{ampP}$ constitute two twogene operons with their respective upstream ORFs (Figures 2A and 2B). PA4219 (ampO) overlaps the first seven base pairs of ampP (Figure 2A). AmpO is a putative seven-transmembrane protein with a strong lipoprotein signal peptide that has a potential cleavage site between amino acids 18 and 19 [23]. The $a m p G$ gene is located 43 bp downstream from PA4392 (ampF), which encodes a putative protein with a DNA-protein cysteine methyltransferase domain (Figure 2B). The function of this domain remains unknown. No lipoprotein signal was detected in AmpF.

To determine if $a m p G$ and ampP constitute two-gene operons with their upstream ORFs, RNA isolated from PAO1 was analyzed by reverse transcription polymerase chain reaction $(\mathrm{PCR})$ using primers flanking the intergenic (ampF-ampG) (Figure 3A) and the overlapping 
E. coliAmpG - - - MS S QYLR I FOOPRSA ILLILGFASGLPLALT S GTLOAWM TVENI DLKT I GFFS LVG PA4218(AMPP) MTQQSWREALVAYKSPASLALLLLGFAAGLPTMLVFNTLSVWLREAGVARDT I G FASLG PA4392 (AmpG) - . - . - MLELYRHRRLVITLALLYLSQGIPI GLA MDAL P T LLRQDGA PLQALAFLPLVG

E. coliAmpG QAYVFKFLWS PLMDRYT PPF- - - FGRRRGWLLATQILLLVA I A AMG FLEPG-TQLRWMAA PA4218 (AmpP) LVYAFKWVWS PMLDQWR LP FVGRLGRRR SWLVF SQVLIA L GLLGMALCNPQ - $S$ HLPWL IG PA4392 (AmpG) LPWVVK F LWAPWVDNHWSRR - - LGRRR SWILPMQCMVLACLLGLATLGLGVASAGWAVG

E. coliAmpG LAVVIAFCSASQDIV FDAWK T DVL PAEERGAGAAI SVLGYRLGMLVSGGLALWLADKW - PA4218 (AmpP) LALLVAFA SATQDIAIDAYRLEI AEDSRQAALAACYMTGYRAA ILLASAGALILAEWFGS PA4392 (AmpG) LLALA S LA SA TQD IATDGMAAEH F S GELLAKVNAVQ I AGVM I GF FG GGAGSL I LAGHF- -

E. coliAmpG - - - - - - LGW QGMYWLMAALL IPCI I A TLLA PEPT DT IPVPK T LEQAVVA P LRDF F GRNN PA4218 (AmpP) T SLNY S S AWGL TYALFALLILPGLVT SLLI REPA VDVPIHVN P S F D FNHOLL SVLLLL PA4392 (AmpG) - - - - - - GQRT AF LVMACVPLA SLC CVLA LGRGD P H L P PA P AAKAS LLR FLR R PLA P S

E. coliAmpG AWLILLLIVLYKLGDA F AMSLTT TFLIRGVGF DAGEVGVVNKT LGLLATI VGALYGG ILM PA4218(AmpP) ILL ISLPAM TTALLDRAWPRA GLYALLMGI CL S PWGRQQ IR PVRELLATVRR PLLVAARG PA4392 (AmpG) LLA LALLS AMTAVSG FGLSKL- - - YLS DAGWALQDIGR LGMS GGLVTVF LGCGGGAWLV

E. coliAmpG QRL SLFR- - - - - - - ALLTFGI LQGAS NAGYWLLS I T R KHLYSMGAAVF F ENLCGGMG T PA4218 (AmpP) KEVPLFDFVHQAVSVMVLIILLVTVTAMCRAYYSGAWPRGTLF LLIAASCLSAPGRLLMA PA4392 (AmpG) RRI GLWR- - . - . . - - GF AL GVVLA GC SALLWYLQA GRWLALSEGLAWT CVLI GS LA T G

E. coliAmpG SAFVALLMTLCNKSFSATQFALISALSAVGRVYVGPVAGWFVEAHGWS - . . . . . - PA4218(AmpP) PVLTPITEFVQRYRWQALLLLGLISTYRLSDTVMGVMAGVFYIDMGFSKEVIASVSKVFG PA4392(AmpG) I T SVA I LTAAMRA GQGGGAG TDVTAVQSTRD LGEMLAS SFLVSLTAQ - - - - . - . - -

E. coliAmpG - - - - - - PA4218(AmpP) VLMTLIGAAAGGVLIARFSILSILFIGGAASAATNLLFAMLAQLGPLHAEALPSHDVISL PA4392(AmpG) -

E. coliAmpG AAAVPGLILLLVCROTLEYTRVNDNF ISRTAYPAGYAFAMWTLAAGVSLLAVWLLL- - PA4218(AmpP) VMALEP HVLMLVMTITLDNFSGGLAASA FVAYLSSLTNLKFSATQYAMLS S TMLLLPRF I PA4392 (AmpG) LTGSALAVLALLLALRLQA GEGRGEWKGRAEEA

E. coliAmpG - - - - - L TMDALD L THFS F L PALLEVGVLVA L S GVVLG GL LDYLALRKTHLT - - PA4218(AmpP) GGYSGTMVESLGYEHFFYVTAVMGI P TLLLI GWLWLRRAPS S AAPA P GHQNAEQH PA4392 (AmpG) - . . . - .

Figure 1 Alignment of E. coli AmpG, PA4218 and PA4393. The primary sequence of E. coli AmpG, PA4218 (AmpP) and PA4393 (AmpG) were used as an input to M-Coffee, which combines multiple sequence alignments using the T-Coffee platform $[45,46]$. Identical and similar amino acids were shaded black and gray, respectively, using BOXSHADE.

$(a m p O-a m p P)$ region (Figure $3 \mathrm{~B})$. The expected amplicon sizes are 136 and 158 bp for the ampF-G junction and $\operatorname{ampO}-P$ junction, respectively [23]. As expected, amplification was observed with genomic DNA (Figures 3A and 3B, Lane 3). In the RNA analyses, PCR products were observed in reverse transcription PCR when the template was prepared in the presence of reverse transcriptase (Figures $3 \mathrm{~A}$ and $3 \mathrm{~B}$, Lane 1), but not in the control reaction when reverse transcriptase was omitted (Figures $3 \mathrm{~A}$ and 3B, Lane 2). This confirms that $a m p O$ and $a m p P$ constitute a two-gene operon and $a m p F$ and $a m p G$ constitute another. In addition, reverse transcriptase real time PCR data is in agreement with $a m p O$ and $a m p P$ belonging to the same operon and $a m p F$ and $a m p G$ comprising another operon (data not shown).
Topology analysis of AmpG and AmpP

The $a m p G$ and $a m p P$ genes encode predicted proteins with 594 and 414 amino acids, isoelectric points of 9.3 and 9.4, and calculated molecular weights of $64.6 \mathrm{kDa}$ and $43.2 \mathrm{kDa}$, respectively. Hydrophobicity plots predict that AmpG has 16 or 14 predicted transmembrane (TM) helices, depending upon the algorithm used and AmpP has 10 [23]. To determine the membrane topology of AmpG and AmpP, phoA or lacZ was cloned downstream of the $\operatorname{amp} G$ and $a m p P$ genes. The 3'-end of the $a m p G$ and $a m p P$ genes were progressively deleted using exonuclease III. At various time-points, the truncated genes were ligated and assayed for PhoA and LacZ activities in E. coli. Clones were also sequenced to determine the reporter and $a m p$ gene junctions. 


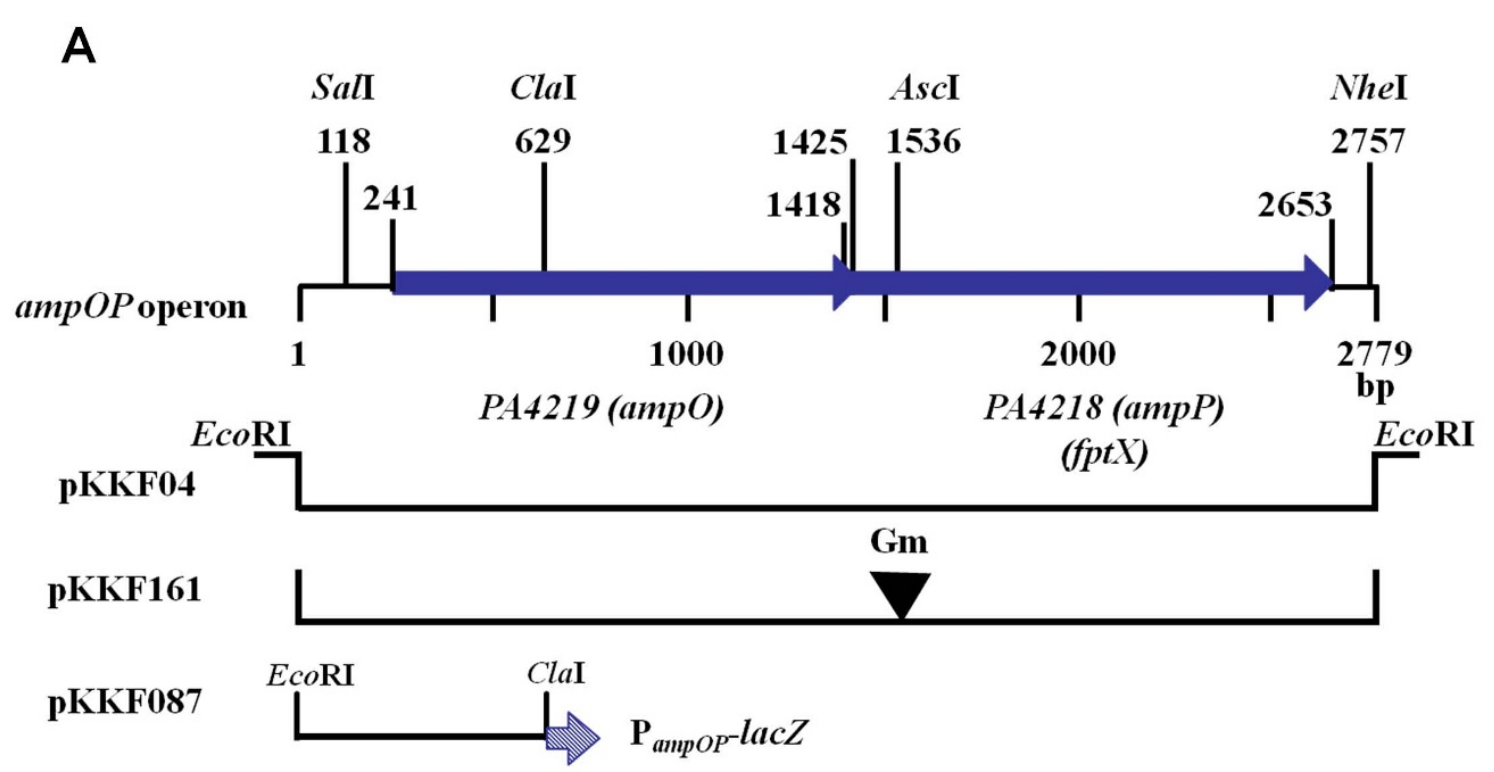

B

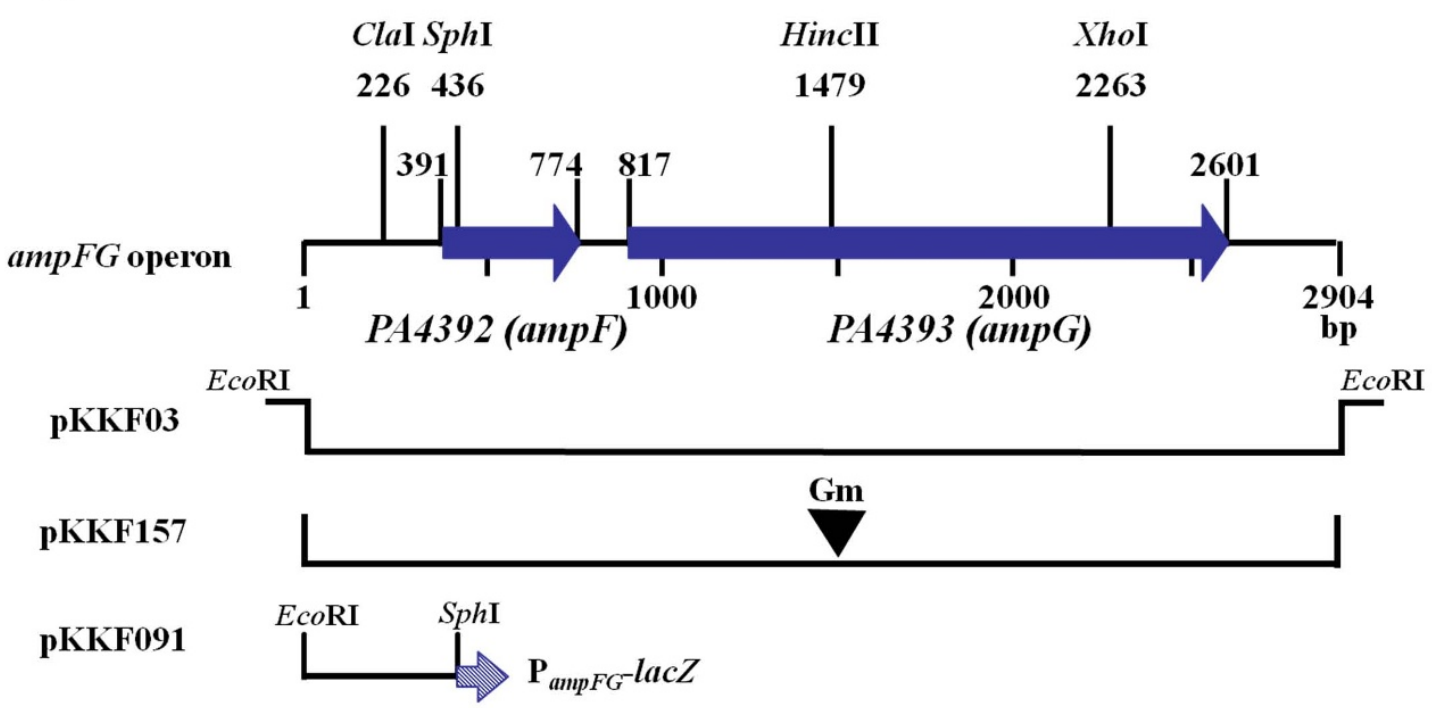

Figure 2 Physical map of the $\operatorname{ampO-ampP}(\mathbf{A})$ and $\boldsymbol{a m p F - a m p G}(\mathbf{B})$ loci. The restriction map is based on PAO1 genome sequence with relevant restriction sites. (A) The 2779-bp ampO-ampP fragment has the PAO1 coordinates of 4721496 to 4724275 . (B) The 2904-bp ampF-ampG fragment corresponds to the PAO1 coordinates of 4921591 to 4924494 . The plasmids pKKF03 and pKKF04 are derivatives of pCRII-TOPO

(Invitrogen, CA), whereas pKKF157 and pKKF161 are derivatives of pME6030 [41]. The Gm cassette (black inverted triangle) was inserted into the Hincll and Ascl sites of pKKF03 and pKKF04, respectively.

AmpG fusions at amino acids 80, 146, 221, 290, 368, $438,468,495$, as well as full length were LacZ-positive and PhoA-negative, and fusions at amino acids 51, 185, $255,338,406$, and 540 were PhoA-positive and LacZnegative domains, suggesting that AmpG has only 14 TM helices (Figures $4 \mathrm{C}$ and 4D). AmpP fusions at amino acids 80, 170, 248, 308, 400 as well as full length were LacZ-positive and PhoA-negative, and fusions at amino acids 38, 120, 195, 278, and 360 were LacZ-negative and PhoA-positive, consistent with $10 \mathrm{TM}$ domains (Figures $4 \mathrm{~A}$ and $4 \mathrm{~B}$ ).

$\beta$-lactamase activity in strains containing mutations in $a m p G$ and $a m p P$

The failure to induce $C$. freundii $a m p C$ in the absence of E. coli ampG suggested that AmpG is essential for 
A

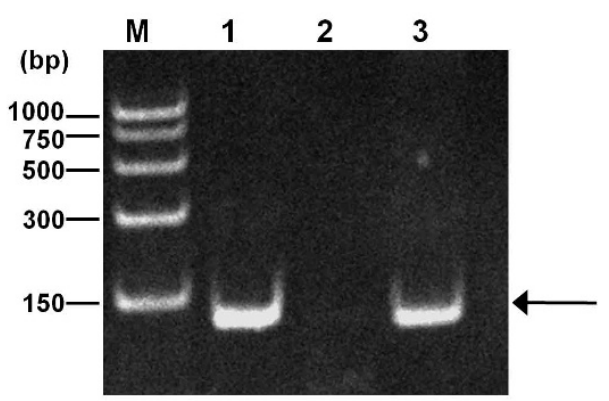

B

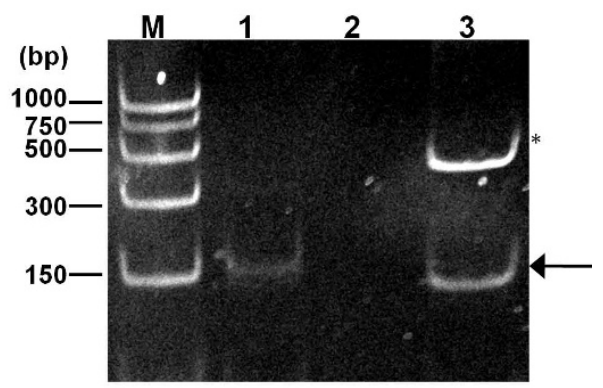

Figure 3 PCR analysis of $a m p F G$ and ampOP operon CDNA. Polyacrylamide gel electrophoresis of PCR products of the junctions of the ampOP and ampFG operons. (A) PCR with primers PA4392_3junctionRTF and PA4392_3junctionRTR to amplify the PA4392 - PA4393 intergenic region. (B) PCR with primers PA4218_9junctionRTF and PA4218_9junctionRTR to amplify the PA4392 - PA4393 intergenic region. (Panels A and B) Lane M: PCR markers (Promega, Madison, WI). Lane 1, CDNA reaction performed with PAO1 RNA, the appropriate buffer and Superscript RT III. Lane 2, cDNA reaction performed with PAO1 RNA, the appropriate buffer without Superscript RT III. Lane 3, P. aeruginosa genomic DNA. The asterisk indicates a nonspecific product. Arrows indicate junction amplicons.

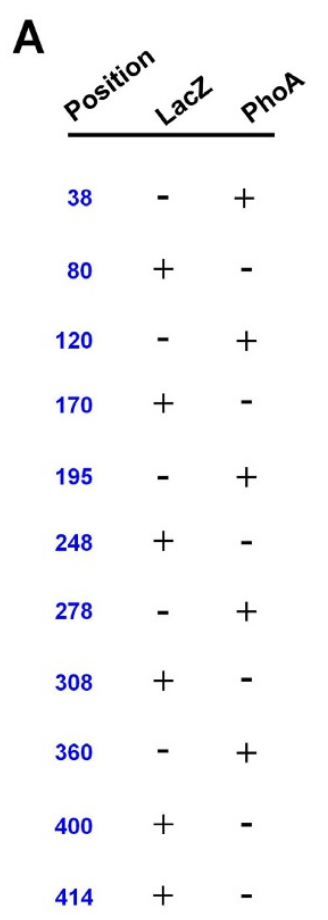

B

Cyto

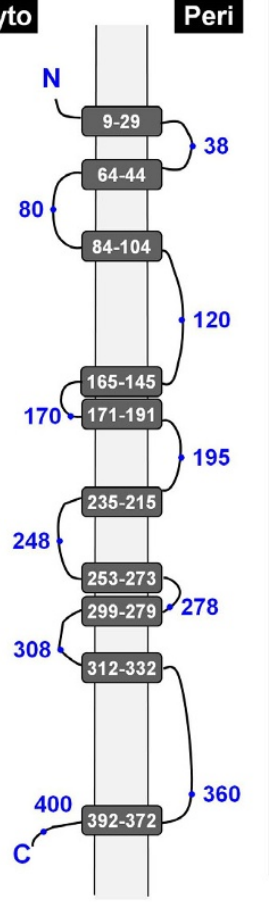

C

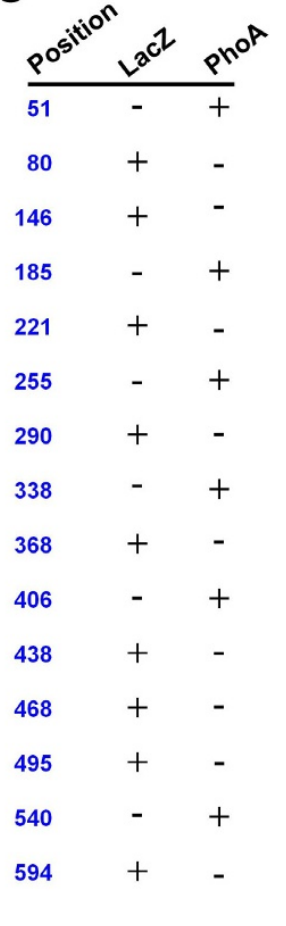

D

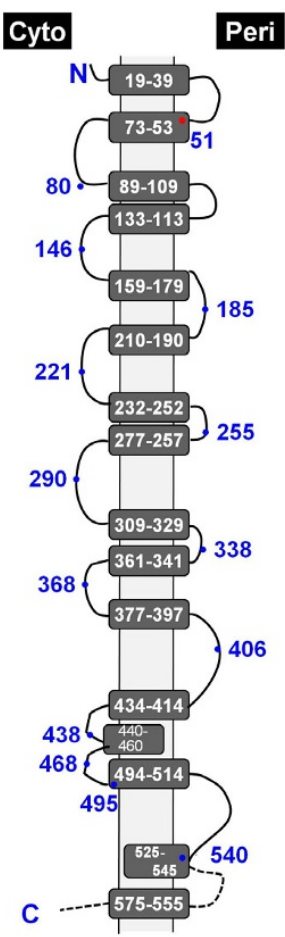

Figure 4 Topology of AmpP and AmpG. The topology of AmpP and AmpG was analyzed by in-frame ampP and ampG fusions to the lacZ and phoA genes, the cytoplasmic and periplasmic markers, respectively. The corresponding points of fusion and qualitative biochemical results of the $\beta$-galactosidase (LacZ) and alkaline phosphatase (PhoA) assays [44] are shown for AmpP (A) and AmpG (C). These results, together with transmembrane domain predictions generated using a Kyte-Doolittle algorithm present in Lasergene 7 (DNASTAR, Madison, WI) were used to predict the topology of AmpP (B) and AmpG (D). Solid lines indicate prediction based upon experimental data, dashed lines indicate regions where more than one possibility exists. Cytoplasm and periplasm are denoted by Cyto and Peri, respectively. Fusion sites are indicated by a dot with the corresponding amino acid number. Putative transmembrane domain boundaries were obtained from Lasergene. 
the induction of chromosomal $\beta$-lactamases $[24,25]$. To ascertain the role of the permeases in $P$. aeruginosa, isogenic amp $G$ and ampP insertional inactivation mutants were constructed in the prototypic $P$. aeruginosa strain PAO1, referred to as PAOampG and PAOampP, respectively. The $\beta$-lactamase activity in the two isogenic mutants, PAOampG and PAOampP, was compared to PAO1. In the absence of $\beta$-lactam antibiotics, all strains showed a basal level of $\beta$-lactamase activity (Table 1 ). Upon challenge with $500 \mu \mathrm{g} / \mathrm{ml}$ of benzyl-penicillin, this level was elevated 10 -fold $(p<0.05)$ in PAO1 (Table 1 ). However, the $\beta$-lactamase activities of PAOampP and PAOampG remained low in the presence of $\beta$-lactam antibiotic, indicating a loss of $\beta$-lactamase induction (Table 1). The loss of inducibility in PAOampG could be partially restored by expressing ampG in trans, whereas the $\beta$-lactamase inducibility of PAOampP was completely recovered when $a m p P$ was supplied in trans (Table 1). Both PAOampP and PAOampG mutants had the other copy of the permease gene intact. These observations suggest that ampG and ampP are individually important members of the $\beta$-lactamase induction system. To confirm that $a m p G$ and ampP play independent roles, cross-complementation of PAOampP with pAmpG, and PAOampG with pAmpP was performed. Similar to the mutants, the cross-complemented strains did not show inducible $\beta$-lactamase activity (Table 1 ).

To further understand the role of $a m p G$ and ampP in $\beta$-lactamase induction, $\beta$-lactamase activity was assayed at different concentrations of benzyl-penicillin in PAO1, PAOampG and PAOampP (Figure 5). Upon encounter with the inducer $(25 \mu \mathrm{g} / \mathrm{ml})$, there was approximately $38 \%$ induction (Figure 5). For strain PAO1, this increase in $\beta$-lactamase activity continued in a dose-dependent manner until the maximum level of $\beta$-lactamase activity was reached when $100 \mu \mathrm{g} / \mathrm{ml}$ of benzyl-penicillin was added (Figure 5). A higher concentration of inducer did not result in a concomitant increase in the expression of the $\beta$-lactamase (Figure 5 and data not shown).

In PAOampG, the initial increase of $\beta$-lactamase activity was observed at $25 \mu \mathrm{g} / \mathrm{ml}$, suggesting that this burst of $\beta$-lactamase production is ampG-independent (Figure 5). However, unlike PAO1, the induction level failed to increase after $25 \mu \mathrm{g} / \mathrm{ml}$ of benzyl-penicillin and even significantly decreased with addition of increased concentrations of benzyl-penicillin (Figure 5).

Mutation of ampP also prevented maximum induction of $\beta$-lactamase, but the defect was not quite as severe as in PAOampG. In PAOampP, the pattern of $\beta$-lactamase induction was very similar to PAO1 at concentrations of benzyl-penicillin up to $50 \mu \mathrm{g} / \mathrm{ml}$ (Figure 5). However, unlike PAO1, addition of benzyl-penicillin at concentrations greater than $50 \mu \mathrm{g} / \mathrm{ml}$ failed to further induce production of $\beta$-lactamases (Figure 5). Thus, low induction is independent of $\operatorname{amp} G$ or $a m p P$. The observation that PAOampP exhibited higher levels of $\beta$-lactamase expression at higher concentrations of benzyl-penicillin may suggest that ampG plays a greater role at higher concentrations of $\beta$-lactam.

Most of the $\beta$-lactamase activity of $P$. aeruginosa can be attributed to AmpC, however, $P$. aeruginosa does contain another chromosomally encoded $\beta$-lactamase, PoxB $[9,26]$. To further analyze if the loss of $\beta$-lactamase induction in the PAOampG and PAOampP strains was due to loss of AmpC function, the $a m p C$ promoter $\left(\mathrm{P}_{a m p C}\right)$ activity was measured in PAO1, $\mathrm{PAO} a m p G$, and PAOampP. As expected, upon treatment with benzylpenicillin, $\mathrm{P}_{\text {ampC }}$-lac $Z$ activity increased approximately 15-fold (Figure 6). Benzyl-penicillin dependent induction of $\mathrm{P}_{a m p C}-$ lacZ was lost in PAOampG or PAOampP (Figure 6).

To further characterize the role of ampG and $a m p P$, the sensitivity of PAO1, PAOampG, and PAOampP to several $\beta$-lactams was determined (Table 2 and data not

Table $1 \beta$-lactamase activity of $P$. aeruginosa PAO1, PAOampG and PAOampP in the absence and presence of $\beta$-lactam

\begin{tabular}{|c|c|c|c|}
\hline \multirow[t]{2}{*}{ Strain and plasmid } & \multirow[t]{2}{*}{ Relevant genotypes (supplement in trans) } & \multicolumn{2}{|c|}{$\beta$-lactamase activity ${ }^{a}$} \\
\hline & & Uninduced & Induced $^{b}$ \\
\hline PAO1 & $a m p G^{+} a m p P^{+}$ & $22.2 \pm 9.7$ & $221.4^{c} \pm 9.2$ \\
\hline PAOampG & $a m p G a m p P^{+}$ & $20.4 \pm 6.2$ & $28.8^{d} \pm 3.3$ \\
\hline PAOampP & $a m p G^{+} a m p P^{-}$ & $4.2 \pm 6.2$ & $32.2^{d} \pm 3.3$ \\
\hline PAOampG/pKKF69 & $a m p G a m p P^{+}\left(a m p G^{+}\right)$ & $8.4 \pm 1.4$ & $87.6 \pm 14.4$ \\
\hline PAOampP/pKKF73 & $a m p G^{+} a m p P^{-}\left(a m p P^{+}\right)$ & $8.8 \pm 1.8$ & $217.9 \pm 35.5$ \\
\hline PAOampG/pKKF73 & $a m p G a m p P^{+}\left(a m p P^{+}\right)$ & $2.1 \pm 2.0$ & $14.4 \pm 1.9$ \\
\hline PAOampP/pKKF69 & $a m p G^{+} a m p P^{-}\left(a m p G^{+}\right)$ & $5.3 \pm 1.9$ & $10.6 \pm 2.7$ \\
\hline
\end{tabular}

${ }^{a}$ Cultures at OD600 of 0.6-0.8 were divided in two. One set was induced with $500 \mu \mathrm{g} / \mathrm{ml}$ benzyl-penicillin for three hours before harvesting. Assays were performed on sonicated lysate using nitrocefin as a chromogenic substrate. One milliunit of $\beta$-lactamase is defined as 1 nanomole of nitrocefin hydrolyzed per minute per microgram of protein. Assays were performed in triplicate.

${ }^{\mathrm{b}}$ Induction was carried out using $500 \mu \mathrm{g} / \mathrm{ml}$ benzyl-penicillin.

c $p<0.05$ compared to uninduced PAO1.

${ }^{\mathrm{d}} p<0.05$ compared to induced PAO1. 


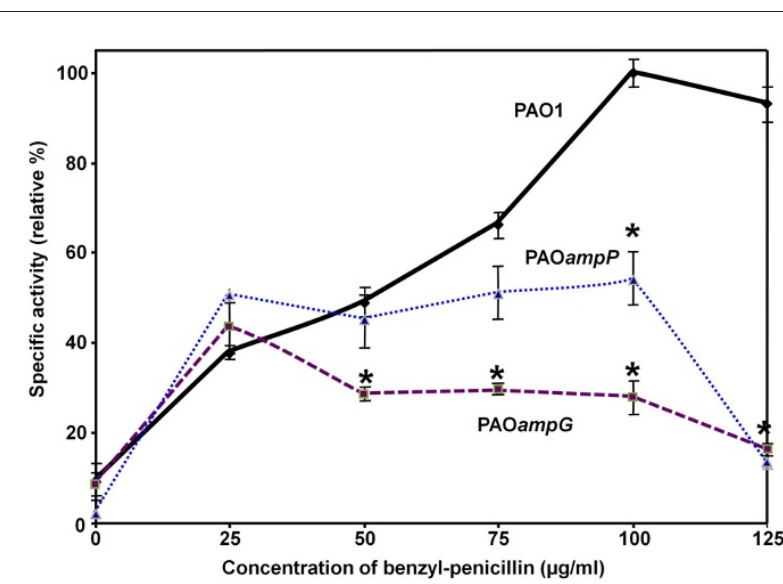

Figure 5 Relative $\beta$-lactamase activity in PAOampP and PAOampG mutants. Assays were performed on the parental PAO1, and the mutants, PAOampP and PAOampG in the presence of benzyl-penicillin at a concentration gradient of 0 to $125 \mu \mathrm{g} / \mathrm{ml}$. Cultures at $\mathrm{OD}_{600}$ of 0.6-0.8 were induced for three hours before harvesting. Assays were performed on sonicated lysate using nitrocefin as a chromogenic substrate. The $\beta$-lactamase activity of PAO1 at $100 \mu \mathrm{g} / \mathrm{ml}$ of benzyl-penicillin was taken as 100\%. Each value is the mean of at least three independent experiments. The asterisk refers to $p$-values of $<0.05$ with respect to PAO1, which were calculated using the two-tailed Student's t-test.

shown). Inactivation of $\operatorname{amp} G$ led to a significant decrease in resistance to amoxicillin ( $>16$-fold) and imipenem ( $>$ seven-fold). No difference was observed with ampicillin/sulbactam, cefaclor, cefepime, oxacillin, piperacillin, piperacillin/tazobactam, or ticaricillin/clavulonic acid (data not shown). Inactivation of ampP in PAO1 did not alter its resistance profile with these $\beta$-lactams (Table 2 and data not shown).

\section{AmpR regulation of $\mathrm{P}_{\text {ampFG }}$ and $\mathrm{P}_{\text {ampoP }}$}

In inducible amp systems, the expression of $\operatorname{ampC}$ is tightly regulated by the transcription factor, AmpR [27]. In order to investigate the role, if any, of $A m p R$ in the regulation of $P$. aeruginosa amp $G$ and ampP, $\mathrm{P}_{\text {ampFG }}$ lac $Z$ and $\mathrm{P}_{\text {ampOP}}$-lacZ promoter fusions were generated and integrated into the chromosome of PAO1 and $\mathrm{PAO} a m p R$ via attB-attP site-specific recombination. These constructs are likely to mimic the chromosomal regulation of the $a m p F G$ and $a m p O P$ operons. In the absence of inducer in PAO1 and PAOampR, there was a detectable basal level of promoter activity (Figure 7). The expression of the $\mathrm{P}_{\text {ampOP-lacZ }}$ promoter fusion was significantly increased in the presence of inducer in the wild-type PAO1, and this induction was lost completely in PAOampR (Figure 7). However, the activity of the $\mathrm{P}_{\text {ampFG-lacZ }}$ promoter fusion was comparable to the basal level in the absence and presence of inducer in PAO1 and PAOampR.

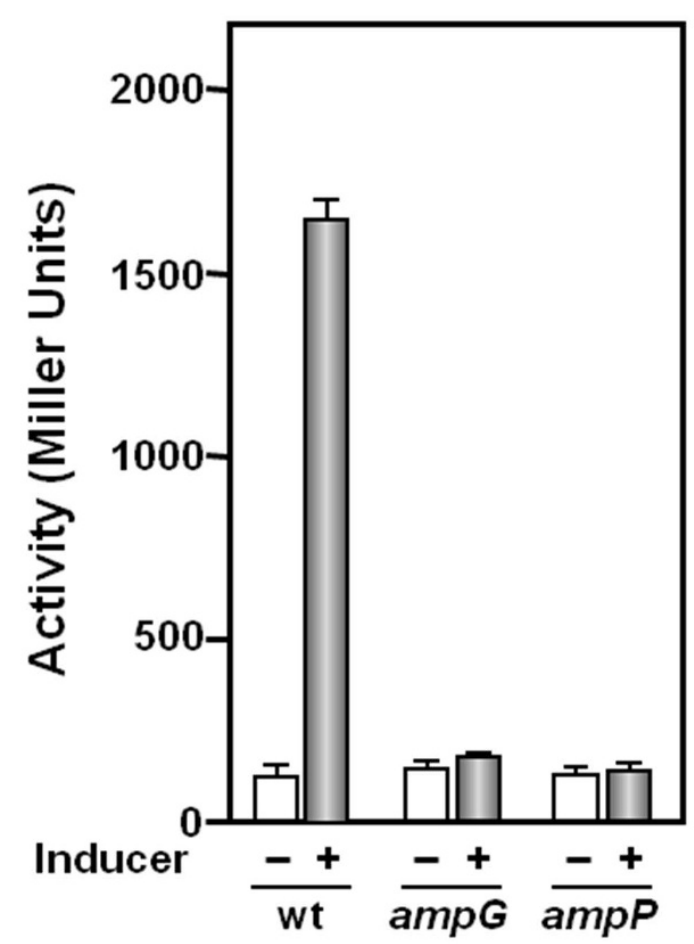

Figure 6 Activity of the $a m p C$ promoter. Promoter activity of the $a m p C$ gene was analyzed using lacZ transcriptional fusions integrated at the att locus of PAO1, PAOampR, PAOampG and PAOampP (see Materials and Methods and text for details). Cells were grown to an $\mathrm{OD}_{600}$ of $0.6-0.8$, at which time cultures were divided into two and one set treated with $100 \mu \mathrm{g} / \mathrm{ml}$ benzylpenicillin. After three hours, cells were harvested and $\beta$ galactosidase activity assayed as described [10]. Each value is the mean of at least three independent experiments.

\section{Autoregulation of the $a m p G$ and $a m p P$ genes}

To determine if $a m p G$ or $a m p P$ affected their own or each other's expression, $\mathrm{P}_{\text {ampFG }}$-lac $Z$ and $\mathrm{P}_{\text {ampOP}}$-lac $Z$ promoter fusions were introduced into the chromosomes of PAOampP and PAOampG. Interestingly, the activity of the $\mathrm{P}_{\text {ampOP}}$-lac $Z$ promoter fusion was significantly de-repressed in PAOampP in the absence and presence of inducer (Figure 7). The activity of the $\mathrm{P}_{a m p F G}-$ lac $Z$ was unchanged in PAOampG in either the absence or presence of benzyl-penicillin. Interestingly,

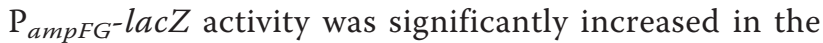
presence, but not absence of $\beta$-lactam in the PAOampP mutant, suggesting $\beta$-lactam-dependent repression of $\mathrm{P}_{\text {amp }}$ by $a m p P$. No change of the promoter activity of the $\operatorname{ampOP}$ operon was observed in the PAOampG mutant.

\section{Discussion}

Members of the Pseudomonadaceae family are intrinsically resistant to $\beta$-lactam antibiotics. Earlier reports 
Table 2 MICs in PAO1, PAOampG and PAOampP strains

\begin{tabular}{ccc}
\hline Strain & \multicolumn{2}{c}{ MIC $(\boldsymbol{\mu g} / \mathbf{m l})$} \\
& Amoxicillin & Imipenem \\
\hline PAO1 & $>256$ & 3 \\
PAOampG & 16 & 0.38 \\
PAOampP & $>256$ & 3 \\
\hline
\end{tabular}

successfully identified $a m p C$, $a m p R, a m p D$, and $a m p E$ as genes involved in the $\beta$-lactamase induction mechanism. However, the question of how chromosomal $\beta$-lactamase is induced remains elusive. This study examines the role of two previously uncharacterized $P$. aeruginosa putative permeases.

\section{$P$. aeruginosa harbors two distinct and independent} AmpG orthologues

In Enterobacteriaceae, besides AmpR, AmpD and AmpE, AmpG has also been implicated in the ampCencoded $\beta$-lactamase induction, acting as a membrane permease that transports 1,6-anhMurNAc-tripeptide and 1,6-anhMurNAc-pentapeptide [17]. In $P$. aeruginosa, two paralogs, $P A 4393 / a m p G$ and $P A 4218 / a m p P$, were found (Figure 1) [28]. Both $a m p G$ and $a m p P$ appear to be one member of two independent two-gene operons (Figures 2 and 3). PFAM analysis of AmpP identifies a Major Facilitator Superfamily (MFS1) domain between amino acids 14 and 346 , in agreement with a role in transport $[23,29,30]$. Upstream from ampP is $P A 4219 /$ amp $O$, a gene that has seven putative transmembrane domains $[23,31]$. Together, these genes form an operon
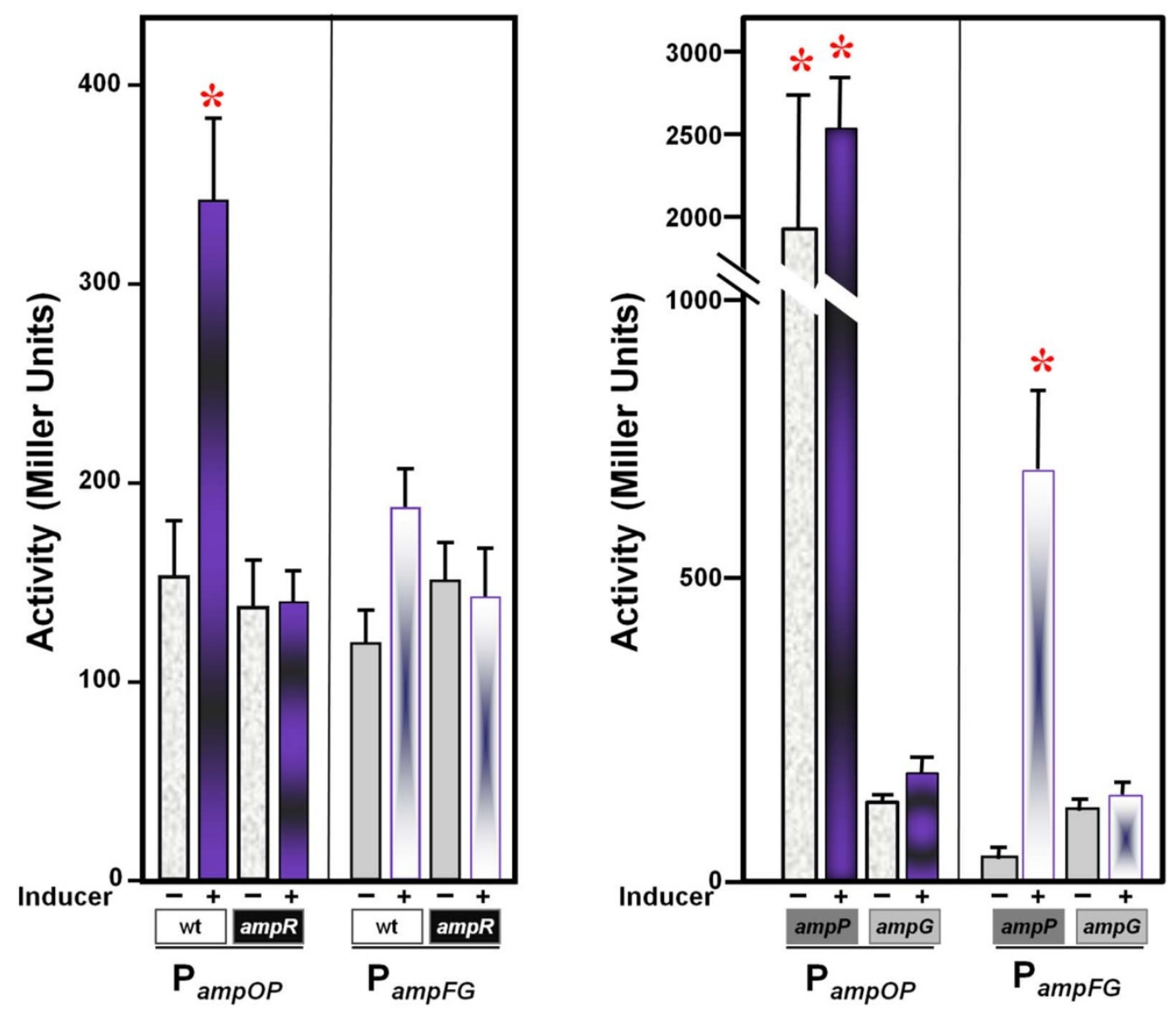

Figure 7 Activity of the ampG and ampP promoters. Promoter activity of the ampG and ampP genes was analyzed using lac $Z$ transcriptional fusions integrated at the att locus of PAO1, PAOampR, PAOampG and PAOampP (see Materials and Methods and text for details). Cells were grown to an $\mathrm{OD}_{600}$ of 0.6 - 0.8, at which time cultures were divided into two and one set treated with $100 \mu \mathrm{g} / \mathrm{ml}$ benzyl-penicillin. After three hours, cells were harvested and $\beta$-galactosidase activity assayed as described [10]. All 16 conditions were assayed at the same time but are divided into two panels for visualization purposes. Each value is the mean of at least three independent experiments. The asterisk refers to $p$ values $<0.05$, which were calculated using the two tailed Student's t-test. 
(Figure 3 ) that is conserved in P. aeruginosa PA14, LES, PACS2, and PA2192 [23,32]. In contrast, PFAM analysis of AmpG does not reveal any significant hits, however, there was an insignificant match to the MFS1 domain $(\mathrm{E}=.00018)[29,30]$. The $a m p G$ gene is downstream from $P A 4392 / a m p F$, which encodes a protein with a putative 6-O-methylguanine-DNA methyltransferase domain $[23,33]$. These two genes also form an operon (Figure 3 ) that is conserved in P. aeruginosa PA14, LES, and PA7 [23].

The topology of the E. coli AmpG permease has been analyzed using $\beta$-lactamase fusion proteins [15]. It was shown that AmpG has ten transmembrane domains with the amino- and carboxyl-termini localized to the cytoplasm [15]. In accordance with roles as transporters, AmpG and AmpP have 14 or 16 (depending upon the algorithm used) and 10, respectively predicted TM domains. PhoA and LacZ fusion analysis corroborates the existence of 14 and 10 TM domains in AmpG and AmpP, respectively (Figure 4). In AmpG, the predicted transmembrane helices between amino acids 440 and 460 and either 525 and 545 or 555 to 575 of PA4393 are likely false positives. AmpG fusions at amino acids 438, 468 and 495 indicate that these amino acids are cytoplasmic (Figure 4), suggesting that if the region between amino acids 440 and 460 is membrane associated, it may be an integral monotopic domain. Similarly, AmpG fusions at residues 495 and 594 are cytoplasmic, while that at 540 is periplasmic, suggesting that if the region between amino acids 525 and 545 is membrane associated, it may be an integral monotopic domain. The fusion data indicates that the carboxyl-termini of both AmpG and AmpP are cytoplasmic (Figure 4 ). Bioinformatic analysis predicts that the amino termini of both proteins are also cytoplasmic. Thus, like E. coli AmpG, both the amino and carboxyl termini would be cytoplasmic [15] (Figure 4).

Consistent with a role in transport, AmpP has an MFS domain [23,30]. The Major Facilitator Superfamily domain is present in approximately one-fourth of all known prokaryotic transport proteins [34]. Interestingly, most MFS proteins have 12 TM domains, while AmpP, like E. coli AmpG, has only 10 [35]. The topology analysis suggests PAO1 AmpG has 14 TM domains. PAO1 AmpG also has an insignificant MFS1 domain. A few MFS proteins have also been shown to have $14 \mathrm{TM}$ domains [29,35].

\section{The ampG and $a m p P$ genes are essential for maximum $\beta$-lactamase induction}

Because of the similarity between AmpG from Enterobacteriaceae and PAO1 AmpG and AmpP, $\beta$-lactamase levels of single ampG and ampP mutant isogenic strains were determined. Although an increase in $\beta$-lactamase activity was observed, neither the ampG nor $a m p P$ mutant strain produced the same level of $\beta$-lactamase in the presence of benzyl-penicillin as PAO1 (Table 1, Figure 5). Moreover, inactivation of ampG or ampP abolishes induction of $\mathrm{P}_{a m p C}$ (Figure 6). This indicates that both $a m p G$ and $a m p P$ are essential for chromosomal $\beta$-lactamase induction. These genes did not cross-complement or exhibit gene dosage effects indicating that they play different roles in the induction pathway (Table 1). These results are consistent with recent data demonstrating that mutation of amp $G$ affects induction of $\beta$-lactamase and failure of ampP to complement an ampG mutation [28]. Furthermore, the analysis using increasing benzyl-penicillin concentrations, shows that $a m p P$ plays an important role at lower inducer concentrations, whereas ampG is crucial at higher concentrations (Figure 5). Mutation of ampG affects PAO1 $\beta$-lactam resistance (Table 2) [28]. Recent studies by Zhang et al., in which deletion of ampG results in increased sensitivity to ampicillin [28], are consistent with results presented here (Table 2). In addition, $\operatorname{amp} G$ inactivation increases imipenem sensitivity (Table 2). Loss of ampP (also referred to as ampGh1) function did not affect $\beta$-lactam sensitivity in either study (Table 2) [28]. AmpP (PA4218) has previously been named FptX due to its homology to RhtX in Sinorhizobium meliloti 2011 [36]. PA4219 does not have a S. meliloti orthologue [36]. Mutation of ampP in a $P$. aeruginosa CDC5 derivative that produces pyochelin but not pyoverdine, resulted in loss of pyochelin utilization [36]. In agreement with a role in pyochelin utilization, ampP is located next to genes involved in pyochelin biosynthesis and transport [23,36]. Thus, the results presented in Table 1 and Figures 5 and 6 demonstrate that ampP is involved in $\beta$-lactamase induction in addition to its previously characterized role in pyochelin utilization [36].

\section{Expression of ampP is induced by $\beta$-lactam addition in the presence of $a m p R$}

Despite the importance of ampG in $\beta$-lactamase induction, little is known about its regulation. E. coli ampG is also the second gene in a two gene operon. Upstream and divergently transcribed from the E. coli ampG operon, is the bolA transcriptional regulator [24]. Expression of bolA is dependent upon RpoS. Previous studies suggest the expression of the E. coli ampG gene is independent of bolA, rpoS or ampD [24]. Neither the $P$. aeruginosa ampG nor ampP gene is located near the bolA locus [23], thus $\mathrm{P}_{a m p F G}$ and $\mathrm{P}_{\text {ampOP }}-$ lac $Z$ transcriptional fusions were integrated into the chromosome of isogenic PAO1 strains to begin to understand ampG and $a m p P$ regulation. 
In light of the requirement of ampG and $a m p P$ for maximum $P$. aeruginosa $\beta$-lactamase induction, it was of interest to determine if expression of either was affected by $\beta$-lactam addition (Table 1 , Figure 5 ). In the absence of antibiotic, $\mathrm{P}_{a m p F G}$ and $\mathrm{P}_{a m p O P}$ were constitutively expressed. Expression of $\mathrm{P}_{a m p O P}$ significantly increased in the presence of inducer, while $\mathrm{P}_{a m p F G}$ did not (Figure 7).

The LysR type transcriptional regulator AmpR induces the expression of the AmpC $\beta$-lactamase in the presence of $\beta$-lactam antibiotics [27]. AmpR also affects the regulation of additional genes involved in $P$. aeruginosa antibiotic resistance and virulence [10]. Insertional inactivation of $a m p R$, did not affect $\mathrm{P}_{a m p F G}-l a c Z$ activity, however, the increase in $\mathrm{P}_{\text {ampOP}}$-lac $Z$ activity previously observed upon $\beta$-lactam addition was lost in the absence of ampR (Figure 7). This indicates that ampP expression is regulated by AmpR. Future analyses will determine if this regulation is direct or indirect.

\section{ampP affects regulation of both its own promoter and that of ampG}

Given that both $\operatorname{amp} G$ and $a m p P$ are required for maximum $\beta$-lactamase expression, both contain structural elements consistent with roles in transport, and the regulation of ampP expression by $\beta$-lactam and $a m p R$, it was feasible that $a m p P$ could contribute to its own expression, perhaps by transporting potential effector molecules for AmpR. Indeed, ampP does appear to inhibit its own expression, as $\mathrm{P}_{a m p O P}$ activity increased ten-fold in PAOampP in the absence, and approximately seven-fold in the presence of $\beta$-lactam (Figure 7). Insertional inactivation of ampP also resulted in increased expression of $\mathrm{P}_{a m p F G}$ in the presence of $\beta$-lactam (Figure 7).

\section{Proposed model for regulation of $\beta$-lactamase induction}

The results presented contribute to what is known concerning $\beta$-lactamase induction in $P$. aeruginosa. It is well established that induction of the expression of the AmpC $\beta$-lactamase is dependent upon AmpR. Although the exact mechanism has not been well characterized in P. aeruginosa, it is believed that the induction is triggered by conversion of AmpR from a repressor to an activator (Figure 8). Evidence from Enterobacteriaceae suggest that this is a result of inhibition of the cell wall remodeling process by $\beta$-lactam antibiotics and subsequent accumulation of intermediates which in turn serve as effector molecules for AmpR. Consistent with previous findings suggesting that AmpR acts as a positive regulator of $a m p$ genes [10], activation of ampP expression required the presence of AmpR and $\beta$-lactam antibiotic (Figure 7). Based upon glycopeptide accumulation studies in other organisms, these findings suggest that the accumulation of 1,6-anhMurNAc-tripeptide and 1,6-anhMurNAc-pentapeptide in the presence of $\beta$-lactam antibiotics activates AmpR that in turn up-regulates the expression of ampP. However, P. aeruginosa appears to use two non-redundant permeases in $\beta$-lactamase induction, suggesting, one may be involved in the import of muramyl peptides and the other in an as yet unknown function. The second permease may be involved in export of muramyl peptides or import of different muramyl peptides. Further studies to determine the identity of these peptides and how they regulate AmpR will be a critical next step in deciphering $\beta$ lactam resistance in $P$. aeruginosa.

The $a m p P$ gene is also auto-regulated via an unknown mechanism. If AmpP performs a similar function as E. coli AmpG, the absence of ampP would result in the accumulation of the periplasmic pool of GlcNAcanhMurNAc peptides or the reduction in the cytoplasmic pool of 1,6-anhMurNAc-tripeptide and 1,6-anhMurNAcpentapeptide alerting the cell that the peptidoglycan recycling process is inhibited. This signalling could result in a positive feedback mechanism that up-regulates the expression of ampP. The accumulation of the periplasmic pool of 1,6-anhMurNAc-tripeptide and 1,6-anhMurNAcpentapeptide in PAOampP is also likely to up-regulate the expression of $P$. aeruginosa PAO1 ampG in the presence of $\beta$-lactam.

Currently, it is not known if PAO1 AmpG and AmpP function similarly to $E$. coli AmpG, however, like ampG, the PAO1 ampG and $a m p P$ are important for $\beta$-lactamase induction [14] (Figure 5, Figure 6, Table 1). Moreover, ampG and $a m p P$ are not functionally redundant as both are required for maximum induction. Their expression is also differentially regulated. An ampP promoterlac $Z$ fusion exhibited increased activity in the presence of $a m p R$ and $\beta$-lactam or the absence of $a m p P$. An $a m p G$ promoter-lac $Z$ fusion was unaffected by the absence or presence of $a m p R$ or $a m p G$. Increased $\beta$-galactosidase activity was observed from the $\operatorname{amp} G$ promoter fusion in the presence of $\beta$-lactam in an ampP mutant (Figure 7). It is not known if this is dependent upon $a m p R$, related to an $a m p R$-independent function of ampP in $\beta$-lactamase induction or the function of $a m p P$ in pyochelin utilization.

\section{Conclusions}

$P$. aeruginosa appears to have two ampG paralogs, $a m p G$ and $a m p P$, which encode proteins with 14 and 10 transmembrane domains. Both are required for maximum induction of chromosomal $\beta$-lactamase and induction of the $a m p C$ promoter. Expression of ampP did not restore maximum $\beta$-lactamase induced activity in an ampG mutation nor did expression of ampG 


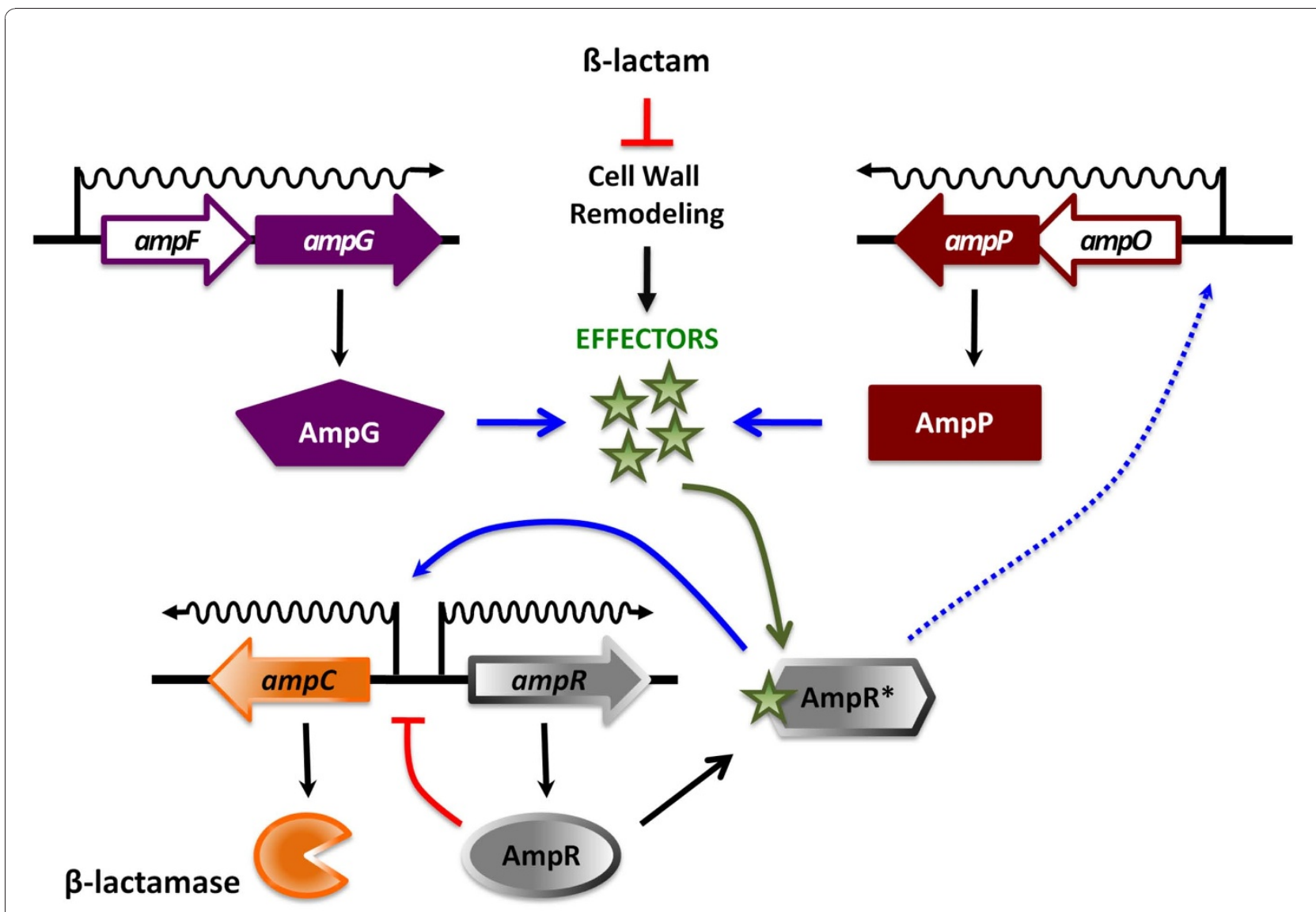

Figure 8 Model for regulation of AmpC $\beta$-lactamase induction by AmpR, AmpP and AmpG in P. aeruginosa. In Enterobacteriaceae as well as P. aerugniosa, the induction of $\beta$-lactamase expression is due to the action of the LysR transcriptional regulator, AmpR. In vitro studies suggest that $A m p R$ can act as either a repressor or an activator, depending upon the presence of different peptidoglycan remodelling intermediates. In this study, it is shown that unlike previously characterized systems, $P$. aeruginosa has two putative AmpG permease paralogs, AmpG and AmpP. Expression of AmpP is inducible by $\beta$-lactam in an ampR-dependent manner. The ampP gene also appears to repress its own expression independent of $\beta$-lactam through an unknown mechanism. Although not observed to be induced by $\beta$-lactam in a PAO1 background, expression of ampG also appears to be repressed by ampP in the presence of $\beta$-lactam (see text for details).

complement an ampP mutation, indicating that $\operatorname{amp} G$ and $a m p P$ have distinct functions in $\beta$-lactamase regulation. In addition to being autoregulated, $a m p P$ is regulated by AmpR and $\beta$-lactam. ampP is also involved in the regulation of $a m p G$ in the presence of $\beta$-lactam. In summary, the presence of two distinct permeases required for $\beta$-lactamase induction suggests that the $P$. aeruginosa $\beta$-lactamase resistance mechanism is more complex and distinct from the current paradigm.

\section{Methods}

Bacterial strains, plasmids and media

Bacterial strains, plasmids and primers employed in this study are shown in Table 3 . E. coli and P. aeruginosa were routinely cultured in Luria-Bertani medium (10 g tryptone, 5 g yeast extract, $5 \mathrm{~g} \mathrm{NaCl}$, per liter). Pseudomonas Isolation Agar (PIA, Difco) was used in triparental mating experiments. Mueller-Hinton agar (Difco) was used in E-test experiments. Antibiotics, when used, were at the following concentrations (per liter) unless indicated otherwise: ampicillin (Ap) at $50 \mathrm{mg}$, tetracycline (Tc) at $20 \mathrm{mg}$, gentamycin $(\mathrm{Gm})$ at $30 \mathrm{mg}$ for $E$. coli and carbenicillin $(\mathrm{Cb})$ at $300 \mathrm{mg}, \mathrm{Gm}$ at $300 \mathrm{mg}$ and Tc at $60 \mathrm{mg}$ for P. aeruginosa.

\section{DNA manipulations}

Standard procedures in molecular biology were performed as previously described [37].

Insertional inactivation of the ampG and ampP genes A 2904-bp ampG fragment was PCR-amplified from PAO1 genomic DNA using KKF01ampGFor and KKF04ampGRev (Table 3). Similarly, KKF05ampPFor and KKF08ampPRev were used to PCR-amplify a 
Table 3 Bacterial strains, plasmids and primers used in this study

\begin{tabular}{|c|c|c|}
\hline Strains/Plasmids & Genotype & Reference \\
\hline \multicolumn{3}{|l|}{ Escherichia coli } \\
\hline TOP10F' & $\begin{array}{l}\left.\mathrm{F}^{\prime}\left\{\text { \{lacl9, Tn10(Tet }{ }^{\mathrm{R}}\right)\right\} \text { mcrA } \triangle(\text { mrr-hsdRMS-merBC) f80dlacZDM15 } \triangle \text { lacX74 deoR recA1 araD139 } \triangle \text { (ara-leu) } 7697 \\
\left.\text { galU galk rpsL (Str }{ }^{\mathrm{R}}\right) \text { endA1 nupG }\end{array}$ & Invitrogen \\
\hline
\end{tabular}

\section{Pseudomonas aeruginosa}

\begin{tabular}{|c|c|}
\hline PAO1 & Wild-type \\
\hline PKM400 & PAOampP::aacCl; $\mathrm{Gm}^{\mathrm{R}}$ \\
\hline PKM500 & PAOampG::aacCl; Gm ${ }^{R}$ \\
\hline PKM300 & PAOampR::aacCl; Gm ${ }^{R}$ \\
\hline PKM301 & PAOattB::P ampclacZ; $T c^{R}$ \\
\hline РКМ303 & PAOampR::aacCl attB::Pampclacz; $T c^{R} \mathrm{Gm}^{R}$ \\
\hline PKM104 & PAOattB::P ampp-lacZ; $T c^{R}$ \\
\hline PKM105 & PAOattB::P ampG $-l a c Z_{;} T c^{R}$ \\
\hline PKM312 & PAOampR::aacCl attB::P ampG-lacz; $\mathrm{Tc}^{R} \mathrm{Gm}^{\mathrm{R}}$ \\
\hline PKM313 & PAOampR::aacCl attB::P ampp-lacZ; $T^{R} c^{R} m^{R}$ \\
\hline PKM404 & PAOampP:::aacCl attB::Pampp-lacZ; $T c^{R} G m^{R}$ \\
\hline PKM405 & PAOampP:::aacCl attB::PampG $l a c Z ; T^{R} c^{R} m^{R}$ \\
\hline PKM504 & PAOampG::aacCl attB::P ampp-lacZ; $T c^{R} G m^{R}$ \\
\hline M505 & PAOampG.:aacCl attB::P ampG-lacZ; Tc ${ }^{R} G m^{R}$ \\
\hline M506 & PAOampG::aaCl attB::P ampclacZ; $T c^{R} G m^{R}$ \\
\hline M507 & PAOampP::aaCl attB::P ampclacZ; $T c^{R} G m^{R}$ \\
\hline
\end{tabular}

[47]

This study

This study

[10]

[10]

[10]

KKF0290, This

study

This study

This study

This study

This study

This study

This study

This study

This study

This study

\section{Plasmids}

pCRII-TOPO

pUCGm

pEX100T

pMF54

pME6030

pRK2013

pTrcphoA

pTrclacZ

pSJ10

pKKF003

pKKF004

pKKF069

pKKF073

pKKF087

pKKF091

pKKF145

pKKF149

pKKF157

pKKF161

pAA0115

PAA0112

PAA0121

pAA1261

pKKF259

pKKF458

pKKF459

pKKF465
$A p^{R}, \mathrm{Km}^{\mathrm{R}}$; ColE1ori lacZ $\alpha$

$A p^{R}, G m^{R} ; p U C 19$ derivative containing gentamycin cassette

$A p^{R}$; sacB oriT

ColE1-SF broad-host replicon

$\mathrm{TC}^{\mathrm{R}}$; oriV pvs1 oriV p15A $_{\text {oriT }}$

$\mathrm{Km}^{\mathrm{R}}$; ColE1ori-Tra (RK2) ${ }^{+}$

$A p^{R}$; low-copy trc promoter expression vector carrying the lacl ${ }^{a}$ and $p h o A$

$A p^{R}$, low-copy trc promoter expression vector carrying the $\operatorname{lac}^{q}{ }^{q}$ and $\operatorname{lac} Z$

$T C^{R} ;$ CTX-lacZ fused with ampC promoter, $P_{\text {amp }}$

$\mathrm{Ap}^{\mathrm{R}}, \mathrm{Km}^{\mathrm{R}}$; pCRII-TOPO with a 2904-bp fragment containing PAO1 coordinates 4921591-4924494 (PA4392$P A 4393 / a m p F-a m p G)$

$\mathrm{Ap}^{\mathrm{R}}, \mathrm{Km}^{\mathrm{R}}$; pCRII-TOPO with a 2779-bp fragment containing PAO1 coordinates 4721496-4724275 (PA4217PA4218/ampO-ampP)

$\mathrm{TC}^{\mathrm{R}}$; pME6030 with a 2904-bp EcoRl flanked fragment containing ampF-ampG

$T_{C} C^{R} ;$ pME6030 with a 2779-bp EcoRl flanked fragment containing ampO-ampP

$\mathrm{TC}^{\mathrm{R}}$; CTX-lacZ fused with ampP promoter, $\mathrm{P}_{\text {ampP }}$

$T c^{R} ; C T X$-lacZ fused with ampG promoter, $P_{a m p G}$

$A p^{R}, G m^{R} ; p C R I I-T O P O$ derivative with ampP::aacCl

$A p^{R}, G m^{R} ;$ pCRII-TOPO derivative with ampG::aacCl

$A p^{R}, G m^{R} ;$ pEX100T derivative with ampG::aacCl

$A p^{R}, \mathrm{Gm}^{\mathrm{R}} ; \mathrm{pEX100T}$ derivative with ampP::aacCl

$A p^{R}$, pTrcphoA with $1.9 \mathrm{~kb}$ Pstl DNA from pMF54 containing stabilization fragment

$A p^{R}, K^{R}$; pCRII-TOPO containing a 1797 ampG PCR product amplified using primers KKF09 and KKF10

$A^{R}$, pAA0115 containing a 1,813 EcoRl fragment containing ampG from pAA0112

$A p^{R} ;$ pAA0121 digested with BamHI and Sall, treated with Klenow and re-ligated to remove an Xbal site. Used as basis for Erase-a-base system

$A p^{R}$; $p$ TrcphoA derivative with a 1797-bp fragment containing PA4393

$A p^{R}$; $p \operatorname{TrcphoA}$ derivative with a 1245-bp fragment containing PA4218

$A p^{R}$; pTrclacZ derivative with a 1797-bp fragment containing PA4393

$A p^{R}$; pTrclacZ derivative with a 1245-bp fragment containing PA4218
Invitrogen

[38]

[39]

[48]

[41]

[40]

[43]

[43]

[10]

This study

This study

This study

This study

This study

This study

This study

This study

This study

This study

This study

This study

This study

This study

This study

This study

This study

This study 
Table 3 Bacterial strains, plasmids and primers used in this study (Continued)

\begin{tabular}{|c|c|c|}
\hline \\
\hline \multicolumn{3}{|c|}{ 5'-TCCAGCTTGACGTCGAGATT-3' } \\
\hline KKF04ampGRev & 5'-AGAACATTCTCCTGGCCATGG-3' & This study \\
\hline KKF05ampPFor & 5'-AACGGCCACGCTAGCAACAC-3' & This study \\
\hline KKF08ampPRev & 5'-GTGGCGCCTGGAGTCTTG-3' & This study \\
\hline KKF09ampG2For & 5'-GGGAATTCCATATGACTCAGCAATCCTGG-3' & This study \\
\hline KKF10ampG2Rev & 5'-GCTCTAGATGCTCGGCGTTCTGGTGT-3' & This study \\
\hline KKF13ampP2For & 5'-TCTAGATCAGGCCTCTTCCGCCCG-3' & This study \\
\hline KKF14ampP2Rev & 5'-ATGCTTGAGCTGTACCGCCA-3' & This study \\
\hline PA4218_9junctionRTF & 5'-ACCTCACCCTGATCCTCTG-3' & This study \\
\hline PA4218_9junctionRTR & 5'-CAGGTAGAGCAACGCCAG-3' & This study \\
\hline PA4392_3junctionRTF & 5'-CAACGACAGGGTGGACATAC-3' & This study \\
\hline PA4392_3junctionRTR & 5'-GAGACTTGTAGGCGACCAG-3' & This study \\
\hline$(\mathrm{NS})_{5}$ RandomPrimer & $5^{\prime}-N S N S N S N S N S-3^{\prime}$ & [49] \\
\hline
\end{tabular}

2779-bp ampP fragment. The ampP and ampG PCR products were cloned into pCRII-TOPO according to the manufacturer's instruction (Invitrogen, CA), generating pKKF04 and pKKF03, respectively. A Gm cassette carrying the aacCI gene was retrieved from pUCGm [38]. The cassette was inserted into the unique HincII and $A s c$ I restriction sites of $a m p P$ and $a m p G$, respectively, creating pKKF145 and pKKF149 (Figure 2). These insertions created a polar mutation in the 5 '-ends of ampP and ampG ORFs in pKKF04 and pKKF03, respectively. Subsequently, the ampP::aacCI and ampG::aacCI from pKKF145 and pKKF149, respectively, were subcloned into the SmaI site of pEX100T [39], a mobilizable suicide plasmid. These plasmids were conjugated into $P$. aeruginosa $\mathrm{PAO} 1$, with a helper strain harboring pRK2013 [40]. The merodiploids, resulting from homologous recombination, were selected with PIA containing $\mathrm{Gm}$. These $\mathrm{Gm}^{\mathrm{R}}$ colonies were then screened for $\mathrm{Gm}$ resistance and $\mathrm{Cb}$ sensitivity by replica plating. The insertions were confirmed by PCR and restriction analysis of the PCR product (data not shown). The PAO1 isogenic strains with defective $a m p P$ and $a m p G$ are henceforth referred to as PAOampP and PAOampG, respectively.

\section{Construction of ampP and ampG complementing plasmids}

Plasmids containing ampP and $a m p G$, pKKF73 and pKKF69, respectively, were generated by inserting the EcoRI fragment with ampP and $a m p G$ from pKKF004 and pKKF003 into a broad-host range, low copy number vector, pME6030 [41]. These were later conjugated into PAOampP and PAOampG for complementation analysis.

\section{Promoter-lacZ fusion constructions}

The putative promoter regions of ampG and $a m p P$ were subcloned from pKKF003 and pKKF004 into pGEMEX-1, respectively, generating pKKF091 ( $\mathrm{P}_{a m p F G^{-}}$

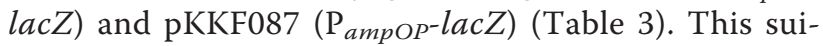
cide vector contained the integration-proficient attP site, which recombines into the chromosomal attB site to generate a single-copy reporter fusion [42]. The resulting clones were mobilized into PAO1 and PAOampR (Table 3). The presence of the chromosomal insertions was confirmed by PCR and restriction analysis of the product.

\section{Topological analysis of AmpP and AmpG}

The topology of AmpP and AmpG were investigated using two markers, phoA and lac $Z$, that function in the periplasm and cytoplasm, respectively. The entire ampP gene was PCR amplified using primers KKF13ampP2For and KKF14ampP2Rev and cloned into pTrcphoA [43]. The entire ampG gene was PCR amplified using primers KKF09ampG2For and KKF10ampG2Rev and cloned into a pTrcphoA plasmid which had been modified by insertion of a broad host range stabilization fragment from pMF54 (Table 3). Both ampG and ampP genes were cloned into pTrclacZ [43]. The erase-a-base system (Promega, WI) was used to generate deletions of the genes from the 3 '-ends. The resulting clones were then sequenced to determine the fusion junctions. The phoA and lac $Z$ activities were determined as previously described [44].

\section{$\beta$-lactamase and $\beta$-galactosidase assays}

$\beta$-lactamase and $\beta$-galactosidase activities were assayed as previously described $[9,10]$. 


\section{Determination of minimal inhibitory concentrations (MICs)}

MICs were determined using E-test strips (Biomerieux, Marcy l'Etoile, France) according to the manufacturer protocols.

\section{Reverse transcription PCR}

For the reverse transcription PCR, RNA was isolated from PAO1 using the RNAeasy mini kit (Qiagen, Valencia, CA) according to the manufacturer protocol. DNA was removed by two sequential 1 hour treatments at $37^{\circ} \mathrm{C}$ with RQ DNaseI (Promega Corporation, Madison, WI) followed by heat inactivation at $65^{\circ} \mathrm{C}$ for $10 \mathrm{~min}$ utes. Synthesis of cDNA was performed with Superscript III reverse transcriptase (RT) (Invitrogen, Carlsbad, CA) using a $(\mathrm{NS})_{5}$ random primer and $5 \mu \mathrm{g}$ RNA according to the manufacturer protocol. A control reaction containing all components except for Superscript III RT was performed in parallel. After cDNA synthesis, RNA was removed by treatment with $0.2 \mathrm{~N} \mathrm{NaOH}$ for 30 minutes at $65^{\circ} \mathrm{C}$. The reactions were neutralized by addition of $0.2 \mathrm{~N} \mathrm{HCl}$ and cDNA was purified using the QIAquick PCR purification kit (Qiagen, Valencia, CA) according to the manufacturer protocol.

PCR reactions to amplify the $a m p F-a m p G$ intergenic region were performed using primers PA4392_3junctionRTF and PA4392_3junctionRTR (Table 3) using GoTaq Flexi (Promega Corporation, Madison, WI). PCR reactions to amplify the ampO-ampP overlapping region were similarly performed with the exception that primers PA4218_9junctionRTF and PA4218_9junctionRTR (Table 3) were used. PCR products were analyzed by electrophoresis on a $10 \%$ polyacrylamide/ $1 \times \mathrm{TBE}$ gel followed by staining with SybrSafe (Invitrogen, Carlsbad, CA).

\section{Acknowledgements}

This work has been supported by NIH-MBRS SCORE (S06 GM08205 and 5SC1Al081376; KM) and Florida International University Teaching

Assistantships to KFK. We are grateful to past and current members of the Mathee crew for their discussions and constructive critique in evaluating the manuscript.

\section{Author details}

${ }^{1}$ Department of Biological Sciences, College of Arts and Sciences, Florida International University, Miami, FL USA. ²Department of Molecular Microbiology and Infectious Diseases, Herbert Wertheim College of Medicine, Florida International University, Miami, FL USA.

\section{Authors' contributions}

KFK identified the $P$. aeruginosa ampG orthologs, PA4218(ampP) and PA4393 $(a m p G)$, constructed the $a m p G$ and $a m p P$ insertional mutants, as well as the lac $Z$ transcriptional fusion strains, performed the $\beta$-lactamase and $\beta$ galactosidase assays and prepared the first draft of the manuscript. AA constructed and assayed the LacZ and PhoA fusions. LS performed the reverse transcription PCR analysis, determined MICs and assisted with data analysis, figure preparation and wrote the submitted draft of the manuscript. KM conceived of the study, participated in its design and execution and helped in manuscript preparation. All authors read and approved the final manuscript.
Received: 8 May 2010 Accepted: 30 December 2010

Published: 30 December 2010

\section{References}

1. Hidron Al, Edwards JR, Patel J, Horan TC, Sievert DM, Pollock DA, Fridkin SK: NHSN annual update: antimicrobial-resistant pathogens associated with healthcare-associated infections: annual summary of data reported to the National Healthcare Safety Network at the Centers for Disease Control and Prevention, 2006-2007. Infect Control Hosp Epidemiol 2008, 29(11):996-1011.

2. Giwercman B, Lambert PA, Rosdahl VT, Shand GH, Hoiby N: Rapid emergence of resistance in Pseudomonas aeruginosa in cystic fibrosis patients due to in-vivo selection of stable partially derepressed betalactamase producing strains. J Antimicrob Chemother 1990, 26(2):247-259.

3. Hancock RE: The bacterial outer membrane as a drug barrier. Trends Microbiol 1997, 5(1):37-42.

4. Wang $Y$, Ha $U$, Zeng $L$, Jin S: Regulation of membrane permeability by a two-component regulatory system in Pseudomonas aeruginosa. Antimicrob Agents Chemother 2003, 47(1):95-101.

5. Oliver A, Canton R, Campo P, Baquero F, Blazquez J: High frequency of hypermutable Pseudomonas aeruginosa in cystic fibrosis lung infection. Science 2000, 288(5469):1251-1254.

6. Costerton JW, Stewart PS, Greenberg EP: Bacterial biofilms: a common cause of persistent infections. Science 1999, 284(5418):1318-1322

7. Fisher JF, Meroueh SO, Mobashery S: Bacterial resistance to beta-lactam antibiotics: compelling opportunism, compelling opportunity. Chem Rev 2005, 105(2):395-424.

8. Lodge JM, Minchin SD, Piddock $\amalg$, Busby JW: Cloning, sequencing and analysis of the structural gene and regulatory region of the Pseudomonas aeruginosa chromosomal ampC beta-lactamase. Biochem J 1990, 272(3):627-631

9. Kong KF, Jayawardena SR, Del Puerto A, Wiehlmann L, Laabs U, Tummler B, Mathee K: Characterization of poxB, a chromosomal-encoded Pseudomonas aeruginosa oxacillinase. Gene 2005, 358:82-92.

10. Kong KF, Jayawardena SR, Indulkar SD, Del Puerto A, Koh CL, Høiby N, Mathee K: Pseudomonas aeruginosa AmpR is a global transcriptional factor that regulates expression of $A m p C$ and PoxB $\beta$-lactamases, proteases, quorum sensing, and other virulence factors. Antimicrob Agents Chemother 2005, 49(11):4567-4575.

11. Jacobs C: Pharmacia Biotech \& Science prize. 1997 grand prize winner. Life in the balance: cell walls and antibiotic resistance. Science 1997, 578(5344):1731-1732.

12. Jacobs C, Frere JM, Normark S: Cytosolic intermediates for cell wall biosynthesis and degradation control inducible beta-lactam resistance in Gram-negative bacteria. Cell 1997, 88(6):823-832.

13. Jacobs C, Huang LJ, Bartowsky E, Normark S, Park JT: Bacterial cell wall recycling provides cytosolic muropeptides as effectors for betalactamase induction. EMBO J 1994, 13(19):4684-4694.

14. Korfmann G, Sanders CC: $a m p G$ is essential for high-level expression of AmpC beta-lactamase in Enterobacter cloacae. Antimicrob Agents Chemother 1989, 33(11):1946-1951.

15. Chahboune A, Decaffmeyer M, Brasseur R, Joris B: Membrane topology of the Escherichia coli AmpG permease required for recycling of cell wall anhydromuropeptides and $\mathrm{AmpC}$ beta-lactamase induction. Antimicrob Agents Chemother 2005, 49(3):1145-1149.

16. Cheng Q, Park JT: Substrate specificity of the AmpG permease required for recycling of cell wall anhydro-muropeptides. J Bacteriol 2002, 184(23):6434-6436

17. Dietz H, Pfeifle D, Wiedemann B: The signal molecule for beta-lactamase induction in Enterobacter cloacae is the anhydromuramyl-pentapeptide. Antimicrob Agents Chemother 1997, 41(10):2113-2120.

18. Cheng Q, Li H, Merdek K, Park JT: Molecular characterization of the beta -N-acetylglucosaminidase of Escherichia coli and its role in cell wall recycling. J Bacteriol 2000, 182(17):4836-4840.

19. Park JT, Uehara T: How bacteria consume their own exoskeletons (turnover and recycling of cell wall peptidoglycan). Microbiol Mol Biol Rev 2008, 72(2):211-227.

20. Altschul SF, Gish W, Miller W, Myers EW, Lipman DJ: Basic local alignment search tool. J Mol Biol 1990, 215(3):403-410. 
21. Altschul SF, Madden TL, Schaffer AA, Zhang J, Zhang Z, Miller W, Lipman DJ: Gapped BLAST and PSI-BLAST: a new generation of protein database search programs. Nucleic Acids Res 1997, 25(17):3389-3402.

22. Needleman SB, Wunsch CD: A general method applicable to the search for similarities in the amino acid sequence of two proteins. $J \mathrm{Mol} B \mathrm{BiO}$ 1970, 48(3):443-453.

23. Winsor GL, Van Rossum T, Lo R, Khaira B, Whiteside MD, Hancock RE, Brinkman FS: Pseudomonas Genome Database: facilitating user-friendly, comprehensive comparisons of microbial genomes. Nucleic Acids Res 2009, , 37 Database: D483-488.

24. Lindquist S, Weston-Hafer K, Schmidt H, Pul C, Korfmann G, Erickson J, Sanders C, Martin HH, Normark S: AmpG, a signal transducer in chromosomal beta-lactamase induction. Mol Microbiol 1993, 9(4):703-715.

25. Schmidt $\mathrm{H}$, Korfmann $\mathrm{G}$, Barth $\mathrm{H}$, Martin $\mathrm{HH}$ : The signal transducer encoded by ampG is essential for induction of chromosomal AmpC beta-lactamase in Escherichia coli by beta-lactam antibiotics and 'unspecific' inducers. Microbiology 1995, 141(Pt 5):1085-1092.

26. Girlich D, Naas T, Nordmann P: Biochemical characterization of the naturally occurring oxacillinase OXA-50 of Pseudomonas aeruginosa. Antimicrob Agents Chemother 2004, 48(6):2043-2048.

27. Hanson ND, Sanders CC: Regulation of inducible AmpC beta-lactamase expression among Enterobacteriaceae. Curr Pharm Des 1999, 5(11):881-894

28. Zhang Y, Bao Q, Gagnon LA, Huletsky A, Oliver A, Jin S, Langaee T: ampG gene of Pseudomonas aeruginosa and its role in beta-lactamase expression. Antimicrob Agents Chemother 2010, 54(11):4772-4779.

29. Pao SS, Paulsen IT, Saier MH Jr: Major facilitator superfamily. Microbiol Mol Biol Rev 1998, 62(1):1-34.

30. Finn RD, Mistry J, Tate J, Coggill P, Heger A, Pollington JE, Gavin OL, Gunasekaran P, Ceric G, Forslund K, Holm L, Sonnhammer EL, Eddy SR, Bateman A: The Pfam protein families database. Nucleic Acids Res 2010, 38 Database: D211-222.

31. Lewenza S, Gardy JL, Brinkman FS, Hancock RE: Genome-wide identification of Pseudomonas aeruginosa exported proteins using a consensus computational strategy combined with a laboratory-based PhoA fusion screen. Genome Res 2005, 15(2):321-329.

32. Pseudomonas aeruginosa Sequencing Project. [http://www.broad.mit.edu]

33. Moore MH, Gulbis JM, Dodson EJ, Demple B, Moody PC: Crystal structure of a suicidal DNA repair protein: the Ada O6-methylguanine-DNA methyltransferase from E. coli. EMBO J 1994, 13(7):1495-1501.

34. Saier MH, Beatty JT, Goffeau A, Harley KT, Heijne WH, Huang SC, Jack DL, Jahn PS, Lew K, Liu J, Pao SS, Paulsen IT, Tseng TT, Virk PS: The major facilitator superfamily. J Mol Microbiol Biotechnol 1999, 1(2):257-279.

35. Law CJ, Maloney PC, Wang DN: Ins and outs of major facilitator superfamily antiporters. Annu Rev Microbiol 2008, 62:289-305.

36. Cuiv PO, Clarke P, Lynch D, O'Connell M: Identification of rht X and fptX, novel genes encoding proteins that show homology and function in the utilization of the siderophores rhizobactin 1021 by Sinorhizobium meliloti and pyochelin by Pseudomonas aeruginosa, respectively. J Bacteriol 2004, 186(10):2996-3005.

37. Ausubel FM, Brent R, Kingston RE, Moore DD, Seidman JG, Smith JA, Struhl K: Short protocols in molecular biology. John Wiley \& Sons, Inc; 4 1999.

38. Schweizer HP: Small broad-host-range gentamycin resistance gene cassettes for site-specific insertion and deletion mutagenesis. Biotechniques 1993, 15(5):831-834

39. Schweizer HP, Hoang TT: An improved system for gene replacement and xyIE fusion analysis in Pseudomonas aeruginosa. Gene 1995, 158(1):15-22

40. Figurski DH, Helinski DR: Replication of an origin-containing derivative of plasmid RK2 dependent on a plasmid function provided in trans. Proc Natl Acad Sci USA 1979, 76(4):1648-1652.

41. Heeb S, Itoh Y, Nishijyo T, Schnider U, Keel C, Wade J, Walsh U, O'Gara F, Haas D: Small, stable shuttle vectors based on the minimal pVS1 replicon for use in Gram-negative, plant-associated bacteria. Mol Plant Microbe Interact 2000, 13(2):232-237.

42. Becher A, Schweizer HP: Integration-proficient Pseudomonas aeruginosa vectors for isolation of single-copy chromosomal lacZ and lux gene fusions. Biotechniques 2000, 29(5):948-950.

43. Blank TE, Donnenberg MS: Novel topology of BfpE, a cytoplasmic membrane protein required for type IV fimbrial biogenesis in enteropathogenic Escherichia coli. J Bacteriol 2001, 183(15):4435-4450.
44. Mathee K, McPherson CJ, Ohman DE: Posttranslational control of the algT (algU)-encoded sigma22 for expression of the alginate regulon in Pseudomonas aeruginosa and localization of its antagonist proteins MucA and MucB (AlgN). J Bacteriol 1997, 179(11):3711-3720.

45. Moretti S, Armougom F, Wallace IM, Higgins DG, Jongeneel CV, Notredame C: The M-Coffee web server: a meta-method for computing multiple sequence alignments by combining alternative alignment methods. Nucleic Acids Res 2007, , 35 Web Server: W645-648.

46. Wallace IM, O'Sullivan O, Higgins DG, Notredame C: M-Coffee: combining multiple sequence alignment methods with T-Coffee. Nucleic Acids Res 2006, 34(6):1692-1699.

47. Holloway BW, Krishnapillai V, Morgan AF: Chromosomal genetics of Pseudomonas. Microbiol Rev 1979, 43(1):73-102.

48. Franklin MJ, Chitnis CE, Gacesa P, Sonesson A, White DC, Ohman DE: Pseudomonas aeruginosa AlgG is a polymer level alginate C5mannuronan epimerase. J Bacteriol 1994, 176(7):1821-1830.

49. Palmer KL, Brown SA, Whiteley M: Membrane-bound nitrate reductase is required for anaerobic growth in cystic fibrosis sputum. J Bacteriol 2007, 189(12):4449-4455.

doi:10.1186/1471-2180-10-328

Cite this article as: Kong et al:: Pseudomonas aeruginosa $\beta$-lactamase induction requires two permeases, AmpG and AmpP. BMC Microbiology $201010: 328$

\section{Submit your next manuscript to BioMed Central and take full advantage of:}

- Convenient online submission

- Thorough peer review

- No space constraints or color figure charges

- Immediate publication on acceptance

- Inclusion in PubMed, CAS, Scopus and Google Scholar

- Research which is freely available for redistribution

Submit your manuscript at www.biomedcentral.com/submit
C) Biomed Central 